\title{
Atypical Sjögren presentation of pruriginous rash and mild eosinophilia with minor sicca symptoms: A case report
}

\author{
Noemie Tremblay*, Alexandra Bourque, Jean-Pascal Costa \\ University of Montreal Hospital Center, Montreal, Canada
}

Received: October 19, 2017

Accepted: December 27, 2017 Online Published: January 6, 2018

DOI: $10.5430 /$ crim.v5n1p18

URL: https://doi.org/10.5430/crim.v5n1p18

\begin{abstract}
We report a case of an 82-year-old woman diagnosed with Sjögren syndrome on the basis of salivary gland biopsy and anti-Ro/SSA antibodies who presented to our institution with a pruriginous maculopapular pancorporeal rash with diffuse eosinophilic infiltrate and mild eosinophilia. Sicca symptoms were mild. Cutaneous and hematologic findings resolved spontaneously within 4 weeks, the rash did not respond to antihistamines but was mildly attenuated by topical corticosteroid. Both the rash and hypereosinophilia greatly improved while the patient received oral corticosteroids for a contrast medium allergy before a CT-scan. When the patient was diagnosed with SS, hydroxychloroquine treatment was introduced. This case report aims to raise awareness of the possibility of such a clinical presentation, considering the known increased risk of non-hodgkin lymphoma associated with Sjögren syndrome estimated to $5 \%-10 \%$ lifetime risk which is 5 to 44 times higher than that of the general population.
\end{abstract}

Key Words: Sjögren, DRESS, Eosinophilia, Rash, Dermatology

\section{INTRODUCTION}

Typical Sjögren syndrome presentation includes xerostomia and xerophtalmia as a result of exocrine gland infiltration by $\mathrm{T}$ and B lymphocytes. Pro-inflammatory cytokines and lympho-attractive chemokines maintain the active autoimmune process and lead to epithelial, ductal and acinar cell apoptosis thereby reducing exocrine activity and, typically, inducing gland hypertrophy. ${ }^{[1]}$

Hyper-reactive B lymphocytes produce auto-antibodies directed against non-organ specific antigens, mainly antiRo/SSA, anti-La/SSB, ANA and rheumatoid factor. A third of SS cases present extraglandular manifestations; arthralgia, myalgias, adenopathies, Raynaud's phenomenon, vasculitis, anemia, leukopenia and pulmonary, renal or hepatic involve- ment are amongst the most common. ${ }^{[1,2]}$

We report an unusual case of Sjögren syndrome in which eosinophilia and a pruriginous maculopapular rash were the main clinical features. Cutaneous manifestations of Sjögren syndrome have been reported, although they are not common. They mostly include xeroderma, purpura and annular erythema. ${ }^{[3,4]}$ To the best of our knowledge, this is the first case of eosinophilic maculopapular Sjögren-associated rash. This case report aims to raise awareness of the possibility of such a clinical presentation, considering the known increased risk of non-hodgkin lymphoma associated with Sjögren syndrome estimated to $5 \%-10 \%$ lifetime risk which is 5 to 44 times higher than that of the general population. ${ }^{[1]}$

*Correspondence: Noemie Tremblay; Email: noemietremblay@live.fr; Address: University of Montreal Hospital Center, Montreal, Canada. 


\section{CASe presentation}

An 82-year-old caucasian woman presented to our institution with complaints of pruriginous maculopapular pancorporeal rash, facial edema and slight weight loss. The physical examination showed generalized indurated maculopapular lesions (see Figure 1), lichenified and excoriated, and axillary and cervical palpable adenomegaly. She remained apyretic throughout the hospitalisation. There had been no recent changes in her medication and she had no known atopy history. She reported a similar rash in her early 60's but had not been investigated at that time.

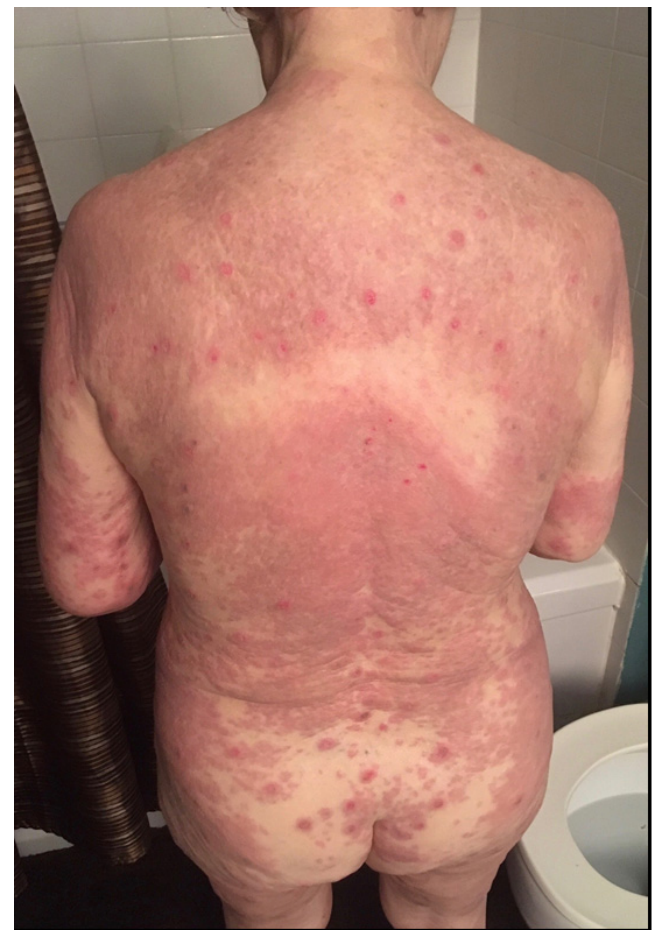

Figure 1. Maculopapular rash

Her laboratory findings at the time were significant for eosinophilia $\left(2.3 \times 10^{9} / \mathrm{L}\right)$ and microcytic anemia $(\mathrm{Hb}$ $96 \mathrm{~g} / \mathrm{L})$. A cutaneous biopsy had been performed and showed a diffuse superficial dermis eosinophilic infiltrate with mild epidermal spongiosis but no primary dermatosis. Blood and stool analysis for parasites was negative. There was no evidence of drug allergic reaction as the IgE count was normal. A pet scan had shown several hypermetabolic adenopathies in the cervical, axillary, inguinal, mediastinal, iliac and paraaortic areas and several discrete cutaneous captation foci, but absence of solid organ neoplasia. A bone marrow biopsy was attempted, but only the sampling of the marrow could be performed due to pain limitation. The marrow showed hyperplasia of the myeloid lineage and high eosinophil content but without morphological anomalies. The rash did not respond to antihistamines but was mildly attenuated by topical

Published by Sciedu Press corticosteroid. The rash and hypereosinophilia had greatly improved with the oral corticosteroid administered in the context of $\mathrm{C}+$ scan and contrast substance allergy, but was not prescribed any further despite the clinical and paraclinical improvement due to myeloproliferative syndrome suspicion. Myeloproliferative or lymphoproliferative syndromes were also considered but the axillary lymph node biopsy result as well as the normal tryptase, JAK 2 and BCR-ABL levels rendered this diagnosis less likely. Eosinophilia evokes parasitic infections which were ruled out through negative anti-strongyloidosis antibodies, stool ova and parasites test, toxocera, trichinosis and quantiferon serology.

Later in her hospitalisation, the patient complained of mild xerostomia without xerophtalmia. A complete rhumatological work-up was requested and showed positive ANA (1/640 granular, 1/160 diffuse), and positive Anti-Ro/SSA antibodies. There were no increase in ESR or CRP. LDH were increased to $324 \mathrm{U} / \mathrm{L}$. Other antibodies and connective tissue disorder markers (anti-LA, anti-ENA, ANCA, anti-sm, anti-DNA, anti-CCP, RF, low $\mathrm{C} 3 / \mathrm{C} 4$ ) were negative. Immunoglobulin levels were normal. A salivary gland biopsy was performed.

Pathology analysis of the salivary gland biopsy showed four perivascular or periductal lymphocyte foci in a $4 \mathrm{~mm}^{2}$ surface, which is the histopathological criteria for Sjögren syndrome. She was diagnosed with Sjögren syndrome on the basis of the salivary gland biopsy, anti-Ro/SSA antibodies and xerostomia. After her hospital discharge, she was treated with hydroxychloroquine. Bone marrow biopsy has not been completed as the diagnosis is established and as normalization of eosinophilic count and cutaneous findings have resolved.

\section{Discussion}

A variety of genetic and non-genetic factors are involved in the pathogenesis of SS and its clinical and paraclinical features are diverse. Eosinophilia has been previously reported in Sjögren syndrome although it is not a typical feature. Secondary polyclonal eosinophilia is most commonly found in high IL-5 contexts such as allergic reactions, helminthic infections and some lymphomas and malignant adenocarcinomas. ${ }^{[5]}$ Pathophysiological explanation for eosinophilia in connective tissue disorders is not fully understood but is attributed to Th2 lymphocytes stimulation leading to higher eosinophilopoietic and anti-apoptotic cytokines levels. ${ }^{[6,7]}$

Cutaneous findings in SS have been described in various instances, but mostly consist of xerose, vasculitis-attributable purpura and annular erythema. ${ }^{[3,4]}$ Diffuse superficial dermis eosinophilic infiltrate can also be seen in atopic dermati- 
tis, eosinophilic panniculitis and other dermatologic conditions ${ }^{[8]}$ but has not been described in Sjögren syndrome.

The eosinophilia and cutaneous lesions being the most predominant features of the case, the diagnosis of SS was strenuous. Systemic allergic reaction or DRESS syndrome were initially suspected but no new medication had been introduced for 2 years and IgE levels were within normal ranges. Chlamydia, mycoplasma, hepatitis, EBV, CMV, Parvovirus B19 and HHV-6 serologies were not performed at the time to rule out viral reactivation induced DRESS syndrome. The lymphocytic infiltration seen in the salivary gland biopsy revealed the patient suffered from SS, as it is the gold standard for SS diagnosis. ${ }^{[1]}$ A superimposed viral reactivation induced DRESS syndrome cannot be ruled out in this context.

Sjögren syndrome prognosis for this patient is not clear, as different SS clinical manifestations are associated with different evolutions. For instance, it has been recognized that patients with severe sicca syndrome have much greater lymphoma relative risk and that patients with cutaneous vasculitis tend to develop life-threatening extraglandular mani- festations. ${ }^{[9]}$ As eosinophilia and non-vasculitis cutaneous manifestations are uncommon features of SS, no data is available on the particular outcomes of this presentation. Studying those outcomes would be pertinent for a better understanding of the prognosis of these patients. There might be many more patients with similar presentation that are not diagnosed with SS as their presentation is atypical.

\section{Conclusion}

In summary, we reported an unusual case of Sjögren syndrome in which eosinophilia and a pruriginous eosinophilicinfiltrated maculopapular rash were the main clinical features. This case report aims to raise awareness of the possibility of such a clinical presentation, considering the known increased risk of non-hodgkin lymphoma associated with Sjögren syndrome and possible later occurrence of life threatening extraglandular manifestations.

\section{CONFLicts OF INTEREST Disclosure}

The authors have declared no conflicts of interest.

\section{REFERENCES}

[1] Longo DL, Fauci AS, Kasper DL, et al. eds. Chapter 324 GoujerotSjögren Syndrome Harrison's Principles of Internal Medicine, 18e New York YO: McGraw-Hill; 2012. 2770-3 p.

[2] Malladi AS, Sack KE, Shiboski SC, et al. Primary Sjögren's syndrome as a systemic disease: a study of participants enrolled in an international Sjögren's syndrome registry. Arthritis Care Res. 2012; 64(6): 911. PMid:22238244 https : //doi .org/10.1002/acr. 21 610

[3] Provost TT, Watson R. Cutaneous manifestations of Sjögren's syndrome. Rheum Dis Clin North Am. 1992; 18(3): 609. PMid:1496164

[4] Brito-Zerón P, Retamozo S, Akasbi M, et al. Annular erythema in primary Sjögren's syndrome: description of 43 non-Asian cases. Lupus. 2014; 23(2): 166. PMid:24326481 https://doi.org/10.1 $177 / 0961203313515764$

[5] Liu Z, Liu Q, Pesce J, et al. Requirements for the development of IL-4-producing $\mathrm{T}$ cells during intestinal nematode infections: what it takes to make a Th2 cell in vivo. Immunol Rev. 2004; 201: 57. PMid:15361233 https://doi.org/10.1111/j.0105-2896.20 $04.00186 . x$
[6] Kang EH, Lee YJ, Hyon JY, et al. Salivary cytokine profiles in primary Sjögren's syndrome differ from those in non-Sjögren sicca in terms of TNF- $\alpha$ levels and Th-1/Th-2 ratios. Clin Exp Rheumatol. 2011; 29(6): 970-6. PMid:22132900

[7] Furst DE. Overview of biologic agents and kinase inhibitors in the rheumatic diseases. Romain PL (Ed) UpToDate. Retrieved April 20th 2017. Available from: www. uptodate.com/contents/over view-of-biologic-agents-and-kinase-inhibitors-in- $t$ he-rheumatic-diseases

[8] Simon D, Wardlaw A, Rothenberg ME. Organ-specific eosinophilic disorders of the skin, lung and gastrointestinal tract. J Allergy Clin Immunol. 2010; 126(1): 3-13. PMid:20392477 https ://doi .org/ $10.1016 / \mathrm{j} \cdot$ jaci.2010.01.055

[9] Voulgarelis M, Dafni UG, Isenberg DA, et al. Malignant lymphoma in primary Sjögren's syndrome: a multicenter, retrospective, clinical study by the European Concerted Action on Sjögren's Syndrome. Arthritis Rheum. 1999; 42(8): 1765. https://doi.org/10.1002/ 1529-0131 (199908) 42:8<1765: : AID-ANR28>3 . 0. CO ; 2-V 